\title{
REGENERATIVE HEALING OF INCISIONAL WOUNDS IN MIDGESTATIONAL MURINE HEARTS IN ORGAN CULTURE
}

Christopher J. Blewett, MD

Robert E. Cilley, MD

H. Paul Ehrlich, PhD

James H. Blackburn II, MD

Peter W. Dillon, MD

Thomas M. Krummel, MD
Objective: Using an organ-culture fetal heart repair model, we explored fetal repair in tissues other than dermis. Methods: Wounded fetal mouse hearts of 14 and 18 days' gestation (term $=20$ days), as well as hearts of 22 days' gestation (newborn), were maintained in serum-free medium. Specimens were fixed at 2,7 , and 11 days and then processed for histologic examination. Small fragments of fetal hearts from all time points were cultured as explants. The migration of cells from the periphery of the explants was compared at day 4 , and the pattern of microfilaments in these cells was assessed. Results: In 14-day hearts $(n=18)$, tissue architecture was rapidly reestablished without an inflammatory response or scarring, constituting regenerative repair. In 18-day hearts $(n=18)$, no reestablishment of muscle fibers or wound closure occurred. In the 22-day explants $(n=12)$ the wounds closed by scarring. Cell migration from 14-day explants was $4.7 \pm 2.3$ ocular units; from 18-day explants, it was $2.6 \pm 1.1$ ocular units; and from 22-day explants, it was $0.9 \pm 0.4$ ocular units. Microfilaments of 14-day cells were arranged at the periphery of the cell consistent with cardiomyocytes. Microfilaments of 18- and 22-day cells were arranged in parallel arrays (stress fibers) that were consistent with fibroblasts. Conclusions: We propose that regenerative healing of 14-day fetal hearts is by the migration of cardiomyocytes. At 18 and 22 days, cardiomyocytes are too differentiated and unable to migrate; hence cell migration is limited to resident fibroblasts, which are deficient at 18 days but sufficient at 21 days to be repaired by the scarring process. (J Thorac Cardiovasc Surg 1997;113: 880-5)
$S^{c}$ carless wound repair has been demonstrated in numerous models of fetal integumentary wound healing. ${ }^{1-4}$ The response of the midgestational fetal dermis to wounding is characterized by a rapid restoration of tissue architecture without an acute inflammatory reaction and with a limited, highly

From the Division of Pediatric Surgery, Department of Surgery, The Pennsylvania State University College of Medicine, The Milton S. Hershey Medical Center, Hershey, $\mathrm{Pa}$.

Partial support from National Institutes of Health grant RO1 GM 41343.

Received for publication August 28, 1996; revisions requested Oct. 8, 1996; revisions received Nov. 6, 1996; accepted for publication Dec. 27, 1996.

Address for reprints: Robert E. Cilley, MD, Division of Pediatric Surgery, Department of Surgery, The Pennsylvania State University, The Milton S. Hershey Medical Center, 500 University Dr., P.O. Box 850, Hershey, PA 17033-0850.

Copyright (C) 1997 by Mosby-Year Book, Inc.

$0022-5223 / 97 \$ 5.00+0 \quad \mathbf{1 2 / 1 / 8 0 1 0 4}$ ordered deposition of collagen fibers to fill the defect. As term approaches, this repair process is converted to the more adultlike wound healing response, with abundant disorganized collagen fibers filling the defect resulting in the formation of a scar.

Research in fetal healing has been largely confined to one organ system-the fetal integument. In addition to the skin, many organ systems figure prominently in the care of the surgical patient and are not limited to but include the cardiovascular, gastrointestinal, and respiratory systems. The structures comprising these systems are frequently injured or subjected to surgical manipulation and incision, and the resulting scar formation in these structures may lead to morbidity and mortality. Some question exists as to whether the unique properties exhibited by the wounded fetal integument are conserved in other organ systems, with evidence supporting both sides of this argument. ${ }^{5-7}$ A recent study has shown that wounds in fetal lungs 

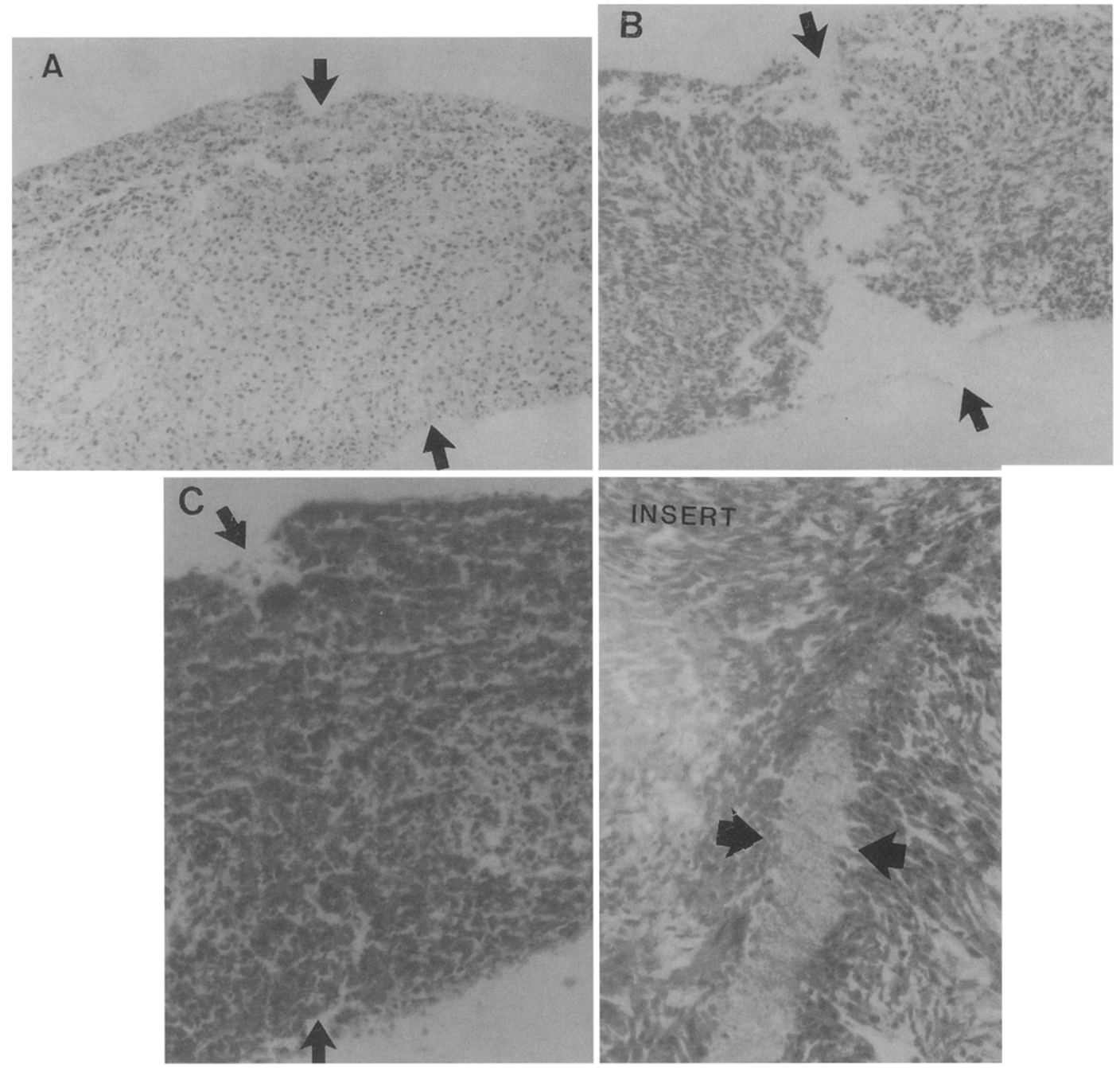

Fig. 1. Photomicrographs of organ cultured hearts. A, Wounded 14-day heart after 2 days in culture stained with hematoxylin and eosin. Arrows identify the wound site, which is otherwise difficult to distinguish from unwounded tissue. B, Cultured 18-day heart harvested 7 days after wounding, stained with trichrome stain. Tissue repair is absent, as demonstrated by the open space between the arrows. C, A wounded 22-day heart explant (wound located between the arrows), which was maintained for 7 days after wounding, stained with trichrome stain. The transition to adult-type healing with scar formation has occurred (as shown in the insert), where newly deposited, poorly organized granulation tissue is deposited.

maintained in organ culture heal in a scarless manner. ${ }^{8}$

Cardiac explants, both embryonic and postnatal, have been successfully maintained in serum-free organ culture for periods exceeding 2 weeks by means of an organ culture technique described by Wildenthal. ${ }^{9}$ Myocardium is often subjected to surgical incision and reapproximation. Scar formation in the heart may have deleterious consequences, primarily because of the development of arrhythmogenic foci. The heart has a large proportion of parenchymal cells relative to fibroblasts. The relative ease of following its viability owing to its contractile activity makes it an excellent organ to study in an organ-culture model of nonintegumentary wound healing.

\section{Materials and methods}

Organ procurement. All animal use was conducted under protocols approved by the sponsoring institution's animal utilization committee. All procedures were in accordance with recommendations of the Panel 
Table I. Summary of histologic analysis from heart explants in culture for 2, 7, or 11 days

\begin{tabular}{cccc}
\hline & & & Duration of culture \\
\cline { 2 - 3 } Gestational period & 2 days & 7 days & 11 days \\
\hline 14 days $(n=18)$ (beating hearts/total) & $6 / 6$ & $6 / 6$ & $6 / 6$ \\
Histology & Healed & Healed & Healed \\
18 days $(n=18)$ (beating hearts/total) & $6 / 6$ & $6 / 6$ & $4 / 6$ \\
Histology & Incomplete healing & Incomplete healing & Incomplete healing \\
22 days $(n=12)$ (beating hearts/total) & $4 / 4$ & $3 / 4$ & $3 / 4$ \\
Histology & Incomplete healing & Collagen scar & Collagen scar \\
\hline
\end{tabular}

Table II. Cell outgrowth from heart explants 4 days in culture

\begin{tabular}{cccc}
\hline $\begin{array}{c}\text { Explant } \\
\text { No. }\end{array}$ & $\begin{array}{c}\text { Explant age } \\
\text { (days) }\end{array}$ & $\begin{array}{c}\text { Circumference } \\
\text { of outgrowth } \\
\text { (degrees) }\end{array}$ & $\begin{array}{c}\text { Cell migration } \\
\text { (ocular units) }\end{array}$ \\
\hline 11 & 14 & $280 \pm 105$ & $4.7 \pm 2.3^{*}$ \\
11 & 18 & $133 \pm 82$ & $2.6 \pm 1.1^{\dagger}$ \\
6 & 22 & $158 \pm 113$ & $0.9 \pm 0.4$ \\
\hline
\end{tabular}

${ }^{*} p=0.01$ compared with 22-day explants.

$\dagger p=0.01$ compared with 14-day explants.

on Euthanasia of the American Veterinary Medical Association and were in compliance with the "Principles of Laboratory Animal Care" formulated by the National Society for Medical Research and the "Guide for the Care and Use of Laboratory Animals" prepared by the Institute of Laboratory Animal Resources and published by the National Institutes of Health (NIH Publication No. 86.23, revised 1985).

Time-dated pregnant CD-1 mice (Charles River Laboratories, Wilmington, Mass.) were killed by cervical dislocation 14 and 18 days after conception (term $=20$ days). Laparotomy and hysterectomy were performed, and the gravid uterus was placed in ice-cold sterile Hanks balanced salt solution (Gibco, Grand Island, N.Y.). Within a laminar flow hood, with the use of sterile technique, individual fetuses were dissected from their amniotic membranes and decapitated. Under a dissecting microscope, a median sternotomy was performed and the thoracic contents were delivered into the field. This allowed identification and dissection of the fetal heart. After removal of attached structures, including the lungs and great vessels, hearts were allowed to equilibrate for 15 to 20 minutes in iced Hanks solution. Additional 2-day-old pups (22 days' gestation) underwent decapitation, after which their hearts were removed in a manner identical to that for the fetal hearts.

Organ culture. Organ culture dishes, $60 \mathrm{~mm} \times 15$ mm, (No. 3037 Falcon, Lincoln Park, N.J.), containing a central well surrounded by an outer well, had their outer well filled with sterile water to maintain a humid environment. Sterile stainless steel wire mesh triangles (Industrial Wire Products, Pasadena, Calif.) were placed over the central well, which was filled with culture medium. The organ culture dishes were placed into $100 \mathrm{~mm} \times 20 \mathrm{~mm}$ Petri dishes (No. 1005 Falcon). The medium consisted of BGJb (Gibco), supplemented with penicillin G $100 \mathrm{U} / \mathrm{ml}$, streptomycin $0.1 \mathrm{mg} / \mathrm{ml}$, amphotericin B $0.25 \mathrm{gm} / \mathrm{ml}$, and sodium ascorbate 1 $\mathrm{mg} / \mathrm{ml}$ at a $\mathrm{pH}$ of 7.4 to 7.5 .

After removal from the fetus or newborn, hearts were placed onto small membrane squares with $0.8 \mu \mathrm{m}$ pores (Millipore, Bedford, Mass.). With the use of a microscalpel, full-thickness linear incisions were created in the right ventricle of the heart directed perpendicular to the long axis of the organ. Wound edges remained well opposed because of surface tension and the splinting action of the underlying membrane. The wounded heart on the membrane was subsequently placed onto the wire screen in the organ culture well and incubated at $37^{\circ} \mathrm{C}$ in $95 \%$ air $/ 5 \%$ carbon dioxide in a water-saturated environment. The culture medium was changed daily, at which time the hearts were examined for signs of contractile activity and visual contamination.

Two, 7, and 11 days after wounding, specimens were fixed in $10 \%$ formalin. After being embedded in paraffin, $5 \mu \mathrm{m}$ sections were stained with hematoxylin and eosin and Masson's trichrome stains.

Delayed wounding. Additional $(n=12)$ 14-day gestation unwounded fetal hearts, harvested as previously described, were placed onto membranes and cultured for 4 days under the conditions described herein. At the time of medium exchange on day 4 after harvest, a fullthickness right ventricular wound was created, as described earlier. The hearts were returned to the incubator, and culture was continued under the same conditions that existed before wounding. Organs were fixed on days 2 and 7 after wounding and were processed for histologic study as described earlier.

Cell migration studies. Murine heart explants were established at 14, 18, and 22 days of gestation by dissecting the heart into small pieces and placing these pieces on $22 \times 22 \mathrm{~mm}$ glass coverslips. The heart explants on the coverslips were then placed in $35 \mathrm{~mm}$ Petri dishes (No. 1008 Falcon) with $2 \mathrm{ml}$ of Dulbecco's modification of Eagle's medium supplemented with $10 \%$ fetal bovine serum and with gentamicin, $10 \mathrm{mg} / \mathrm{ml}$, for 4 days at $37^{\circ} \mathrm{C}$ with $95 \%$ air and $5 \%$ carbon dioxide. On day 4 , the distance migrated by cells from the explant edge was measured with the use of a dissecting microscope with a grid mounted in the eyepiece. The greatest distances traveled were recorded in ocular units along with the circumference of the explant displaying cell migration.

In addition, glass coverslips were fixed for 5 minutes in $4 \%$ paraformaldehyde at a $\mathrm{pH}$ adjusted to 7.4 in 
$\mathrm{Na}_{2} \mathrm{HPO}_{4}, 10 \mathrm{mmol} / \mathrm{L}$, and $\mathrm{NaCl}, 140 \mathrm{mmol} / \mathrm{L}$. The coverslips were washed and the cells were permeabilized in $0.1 \%$ octyphenoxy polyethoxyethanol (Triton X100, Union Carbide Corp., Danbury, Conn.) for 60 seconds before being stained with rhodamine-phalloidin (Molecular Probes, Eugene, Ore.) diluted 1:200. Coverslips were mounted and viewed with an Olympus IMT-2 microscope (Olympus America, Inc., Melville, N.Y.) equipped with fluorescent optics. Photomicrographs were made with Ektachrome ASA 200 film (Eastman Kodak, Rochester, N.Y.).

\section{Results}

Organ culture. All specimens appeared morphologically intact at the time of fixation, without evidence of microbial contamination. During the 11 days of organ culture all of the 14-day hearts continued to beat with an organized contractile rhythm. In all of the 14-day hearts $(n=18)$, tissue architecture was reestablished by 2 days. An inflammatory response was not observed, and collagen fiber deposition did not occur even in hearts cultured for 11 days after wounding (Fig. $1, \mathcal{A}$ ).

Sixteen 18-day hearts of a total of 18 were beating at the times of fixation (2, 7, and 11 days). Histologically, the 18-day specimens lacked an ordered reconstitution of muscle fibers and an incomplete bridging of the wound site by cardiomyocytes. Collagen deposition was not observed in this group by trichrome staining (Fig. 1, $B$ ).

Ten 22-day hearts of a total of 12 were beating at fixation ( 2 and 7 days). These hearts demonstrated repair of their wounds by scarring, with granulation tissue and collagen accumulating at the wound site by 7 days (Fig. 1, C). The insert shows the deposition of granulation tissue in the wound site of the heart. (Table I).

In the 14-day hearts subjected to delayed wounding $(n=12)$, a reconstitution of tissue architecture occurred by 48 hours, the same as in hearts receiving wounds at the time of harvest. No collagen fiber deposition was observed by trichrome staining in this experimental set, even in hearts maintained in culture for 7 days after wounding.

Cell migration studies. Cells grew out from 14day murine heart explants in greater numbers and traveled greater distances than cells from 18- and 22-day murine heart explants (Table II). The groups were compared by means of analysis of variance. Dunnett's test for multiple comparisons was used to determine significance. The 14-day murine heart explants showed cell growth from about three quarters of the circumference of the explant, which

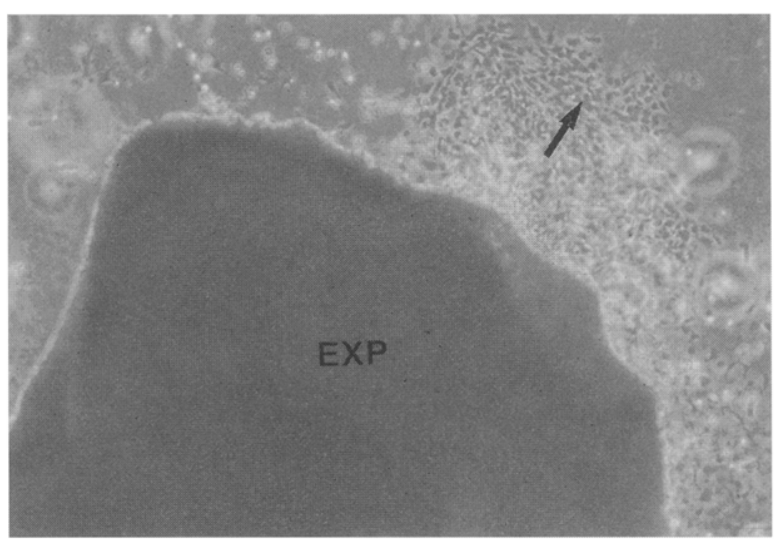

Fig. 2. Outgrowth of cells from murine heart explants. A 14-day murine heart explant showing cell outgrowth from the edge of the explant in the direction of the arrow. Note the distance the cells migrated and the circumference of the explant edge involved in outgrowth.

migrated 1.8 times further than that of the 18-day explants and five times further than that of the 22-day explants. The 18-day and 22-day murine heart explants showed growth from less than half of the circumference of the explant. The migration of cells from the 14-day explants was greater in terms of more cells of the explant involved in the process and the greater distance traveled. The differences were statistically significant (see Table II).

The cells growing out from the 14-day explants tended to migrate as an intact, continuous sheet of cells (Fig. 2). The patterns of cell outgrowth of the 18-day and 22-day explants were more dispersed. Phalloidin-stained explants revealed that the 14-day actin filaments were arranged at the cell periphery, characteristic of cardiomyocytes (Fig. $3, A$ ). In contrast, the 18-day and 22-day migrating cells showed actin filaments as thick bundles of microfilaments (stress fibers) running as parallel arrays in the long axis of the cell, a morphologic pattern typical of fibroblast-like cells (Fig. 3, $B$ ).

\section{Discussion}

Several factors common to nonintegumentary organs suggest that they may exhibit a different response to injury than the skin. Nonintegumentary organs contain parenchymal cells unique to that system, and fibroblasts, the predominant cell type in the integument, comprise a relatively small percentage of the total cells in nonintegumentary organs. In addition, the fetal integument exists in a relatively 

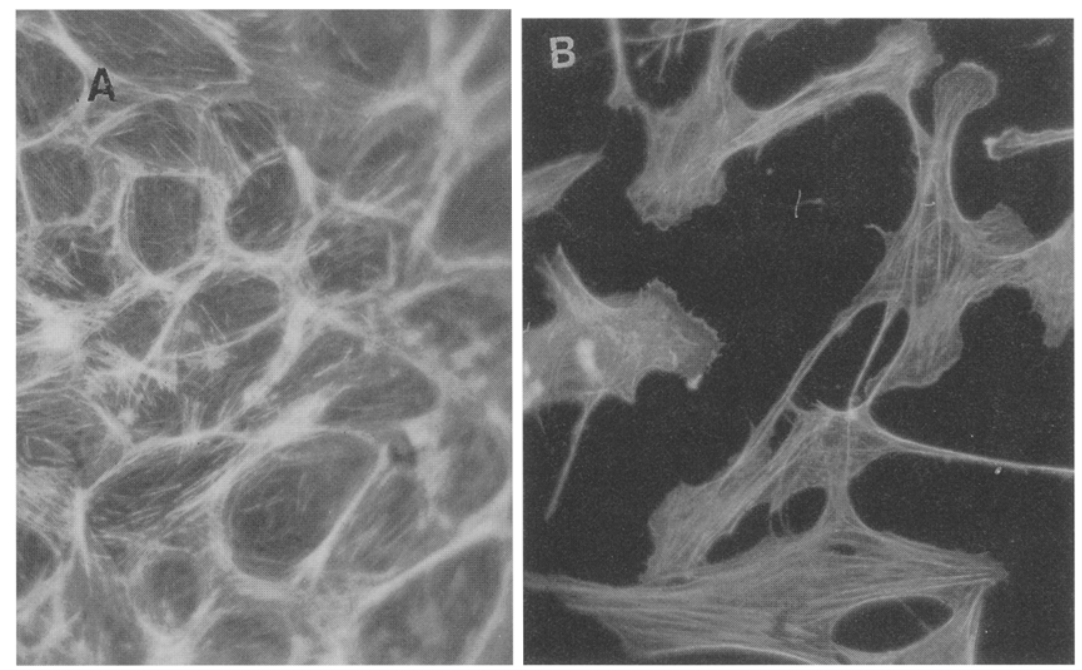

Fig. 3. Actin filament fluorescent staining of 4-day murine heart explant outgrowth. A, A 14-day heart explant showing an intact sheet of cells growing from the edge of the explant with phalloidin-stained actin stress fibers at the periphery of the cells. B, A monolayer of cells growing out from an 18-day murine heart explant showing more randomly arranged cells having actin stress fibers running in parallel arrays along the long axis of the cell. (Original magnifications $\times 120$.)

protected environment where it is not required to function as a barrier against invading microorganisms or other injurious environmental agents. In contrast, other organs such as the heart and kidneys begin to function relatively early in gestation and are critically important to normal embryonic development. With this in mind, we can postulate that such "functioning" organs may demonstrate different properties of healing than the fetal integumentary system. As demonstrated by these experiments, however, midgestational, 14-day murine fetal hearts exhibit the ability to repair incisional wounds in a regenerative fashion, much the same as dermis of a similar age. As in the dermis, this ability is lost as gestation progresses. Furthermore, from the results of the delayed wounding experiments, it appears that this ability of fetal hearts to repair by regeneration depends solely on the local tissue environment. After 4 days of culture with daily media changes, factors transported from the circulation to the wound site would have been dissipated.

The myocardial explant experiments suggest that the regenerative repair of the 14-day wound is accomplished by cardiomyocytes migrating into the defect. It is hypothesized that by gestational day 18 , cardiomyocytes are too differentiated to migrate into the wound and that fibroblast migration into the defect is needed to fill the defect. Fibroblast popu- lations associated with the 18-day heart may be too sparse and their numbers too small to successfully proliferate, migrate, and fill the defect. By day 22 , cardiomyocytes of murine heart explants are also too differentiated to migrate into the defect, but fibroblast populations are sufficient in numbers to migrate, proliferate, and participate in repairing the defect.

The similarities of the ontogeny of cardiac repair in organ culture to that of the dermis suggest that applications of experimental treatments to dermis might be used to modify wound healing in nonintegumentary organs. It has been suggested that a reduction in scarring can be produced by the administration of neutralizing antibodies to transforming growth factor- $\beta .^{10}$ In a similar fashion, Houghton, Keefer, and Krummel ${ }^{11,12}$ have used the limb explant system to reduce scarring in late-gestation limbs by the application of antibodies to plateletderived growth factor and transforming growth factor- $\beta$. A better understanding of the molecular events that control scar formation may allow the wound environment to be manipulated to promote healing by regeneration.

Of particular interest is the ability of the midgestational myocardium to retain a capacity for regenerative healing despite several days in a culture system consisting of a chemically defined serum-free medium. It has recently been shown that fetal 
cardiomyocytes can be transplanted to an adult host, with a satisfactory tolerance of this graft. ${ }^{13}$

The unique properties of wound repair exhibited by fetal dermis seem to be operational in myocardium as well. Further work will be directed toward determining whether other fetal organs demonstrate a similar propensity for scarless repair and whether this unique mechanism of fetal tissue repair can be used to improve the healing of internal organs in both surgical and traumatic injury in the adult patient.

\section{REFERENCES}

1. Somasundaram K, Prathap K. Intra-uterine healing of skin wounds in rabbits foetuses. J Pathol 1970;100:81-6.

2. Burrington JD. Wound healing in the fetal lamb. J Pediatr Surg 1971;6:523-8.

3. Haynes JH, Krummel TM, Flood LC, Cohen IK, Diegelman RF. Fetal skin organ culture. J Invest Surg 1990;3:349-55.

4. Bleacher JC, Adolph VR, Dillon PW, Krummel TM. Isolated fetal mouse limbs: gestational effects on tissue repair in an unperfused system. J Pediatr Surg 1993;28:1312-5.

5. Ris PM, Wray JB. A histological study of fracture healing within the uterus of the rabbit. Clin Orthop Rel Res 1972; $87: 318-21$.
6. al-Qattan MM, Posnick JC, Kant YL, Lin KY, Thorner P. Fetal tendon healing: development of an experimental model. Plast Reconstr Surg 1993;92:1155-60.

7. Longaker MT, Whitby DJ, Jennings RW, Duncan BW, Ferguson MW, Harrison MR, et al. Fetal diaphragmatic wounds heal with scar formation. J Surg Res 1991;50:37585.

8. Blewett CJ, Cilley RE, Ehrlich HP, Dillon PW, Blackburn JH II, Krummel TM. Regenerative healing of incisional wounds in murine fetal lungs maintained in organ culture. J Pediatr Surg 1995;30:945-8.

9. Wildenthal K. Long-term maintenance of spontaneously beating mouse hearts in organ culture. J Appl Physiol 1971;30:153-7.

10. Shah M, Foreman DM, Ferguson MW. Control of scarring in adult wounds by neutralizing antibody to transforming growth factor- $\beta$. Lancet 1992;339:213-4.

11. Houghton PE, Keefer KA, Krummel TM. Platelet derived growth factor neutralizing antibody reduces scar formation in a fetal limb organ culture system. Mol Biol Cell 1993;4:126a.

12. Houghton PE, Keefer KA, Krummel TM. The role of transforming growth factor- $\beta$ in the conversion from "scarless" healing to healing with scar formation. Wound Rep Reg 1993;3:229-36.

13. Soonpaa MH, Koh GY, Klug MG, Field LJ. Formation of nascent intercalated disks between grafted fetal cardiomyocytes and host myocardium. Science 1994;264:98-101.

\section{Bound volumes available to subscribers}

Bound volumes of The Journal of Thoracic and Cardiovascular Surgery are available to subscribers (only) for the 1997 issues from the Publisher, at a cost of $\$ 110.50$ for domestic, $\$ 139.64$ for Canadian, and $\$ 130.50$ for international subscribers for Vol. 113 (January-June) and Vol. 114 (July-December). Shipping charges are included. Each bound volume contains a subject and author index and all advertising is removed. Copies are shipped within 60 days after publication of the last issue of the volume. The binding is durable buckram with the Journal name, volume number, and year stamped in gold on the spine. Payment must accompany all orders. Contact Mosby-Year Book, Inc., Subscription Services, 11830 Westline Industrial Drive, St. Louis, Missouri 63146-3318, USA; phone $800-453-4351$ or $314-453-4351$.

Subscriptions must be in force to qualify. Bound volumes are not available in place of a regular Journal subscription. 